\title{
On some common coupled fixed point results in rectangular b-metric spaces
}

\author{
Feng Gu \\ Institute of Applied Mathematics and Department of Mathematics, Hangzhou Normal University, Hangzhou, Zhejiang 310036, China.
}

Communicated by Y. H. Yao

\begin{abstract}
In this paper, by using the $w$-compatible conditions of mapping pair, we discuss the existence and uniqueness problem of the common coupled fixed point for mappings defined on a set equipped with two rectangular b-metrics. Some new common coupled fixed point theorems are obtained. We also provide illustrative examples in support of our new results. As application, we provide an existence and uniqueness theorem of common solution for a class of nonlinear integral equations by using the obtained new result. The results presented in this paper generalize the well-known comparable results in the literature. (C)2017 All rights reserved.
\end{abstract}

Keywords: Rectangular b-metric space, coupled coincidence point, common coupled fixed point, $w$-compatible mapping pairs. 2010 MSC: $47 \mathrm{H} 10,54 \mathrm{H} 25$.

\section{Introduction and preliminaries}

The concept of rectangular metric space was first introduced by Branciari [6] in 2000. The applications and theories in rectangular metric space have also been extensively studied by many scholars $[2,3,5$, $8,10,12,14-16,18,19,21]$ and they also have proved the common fixed point theorems under Banach contractive conditions and Kannan type contractive conditions in rectangular metric spaces.

In 2015, George et al. [11] introduced the concept of rectangular b-metric space. It is well-known that rectangular b-metric space is an important generalization of usual metric space, b-metric spaces [7], and rectangular metric spaces. Since then, Aydi et al. [4], Ding et al. [9], and Roshan et al. [20] obtained some fixed point and common fixed point results for mappings satisfying different contractive conditions in rectangular b-metric spaces. However, so far, no one discussed coupled fixed point problem in rectangular b-metric space.

The purpose of this paper is to prove some new coupled common fixed point theorems for mappings defined on a set equipped with two b-rectangular metrics. We also provide an existence and uniqueness theorem of solution for a class of nonlinear integral equations by using the obtained result.

Firstly, given some basic notions before introducing some main results.

Definition 1.1 ([7]). Let $X$ be a nonempty set and $s \geqslant 1$ be a given real number. A function $b: X \times X \rightarrow$ $[0, \infty)$ is a b-metric on $X$ if, for all $x, y, z \in X$, the following conditions hold: 
(b1) $b(x, y)=0$ if and only if $x=y$;

(b2) $b(x, y)=b(y, x)$;

(b3) $b(x, z) \leqslant s[b(x, y)+b(y, z)]$ (b-triangular inequality).

In this case, the pair $(X, b)$ is called a $b$-metric space (metric type space).

Definition 1.2 ([6]). Let $X$ be a nonempty set, and let $r: X \times X \rightarrow[0, \infty)$ be a mapping such that for all $x, y \in X$ and all distinct points $u, v \in X$, each distinct from $x$ and $y$ :

(R1) $r(x, y)=0$ if and only if $x=y$;

(R2) $r(x, y)=r(y, x)$;

(R3) $r(x, z) \leqslant r(x, u)+r(u, v)+r(v, z)$ (rectangular inequality).

Then $(X, r)$ is called a rectangular or generalized metric space or Branciari's space.

For properties and definitions of notions in Branciari's spaces see [5].

Definition 1.3 ([11]). Let $X$ be a nonempty set, $s \geqslant 1$ be a given real number and let $r_{b}: X \times X \rightarrow[0, \infty)$ be a mapping such that for all $x, y \in X$ and distinct points $u, v \in X$, each distinct from $x$ and $y$ :

$(\mathrm{Rb} 1) \mathrm{r}_{\mathrm{b}}(x, y)=0$ if and only if $x=y$;

$(\mathrm{Rb} 2) \mathrm{r}_{\mathrm{b}}(x, y)=\mathrm{r}_{\mathrm{b}}(\mathrm{y}, \mathrm{x})$;

(Rb3) $r_{b}(x, z) \leqslant s\left[r_{b}(x, u)+r_{b}(u, v)+r_{b}(v, z)\right]$ (b-rectangular inequality).

Then $\left(X, r_{b}\right)$ is called a rectangular b-metric space (RbMS) or a generalized b-metric space (b-g.m.s). Moreover, $s \geqslant 1$ is the coefficient of rectangular $b$-metric space $X$.

Note that every metric space is a rectangular metric space and every rectangular metric space is a rectangular b-metric space (with coefficient $s=1$ ). However the converse is not necessarily true ([11, Examples 2.4 and 2.5]). Also, every metric space is a b-metric space (metric type space) and every bmetric space is a rectangular b-metric space (not necessarily with the same coefficient).

Note also that every b-metric space with coefficient $s$ is a rectangular b-metric space with coefficient $s^{2}$ but the converse is not necessarily true ([11, Example 2.7]).

Definition $1.4([11])$. Let $\left(X, r_{b}\right)$ be a rectangular b-metric space, $\left\{x_{n}\right\}$ be a sequence in $X$, and $x \in X$. Then

(a) the sequence $\left\{x_{n}\right\}$ is said to be convergent in $X$ and converges to $x$, if for every $\epsilon>0$ there exists $n_{0} \in \mathbb{N}$ such that $r_{b}\left(x_{n}, x\right)<\epsilon$ for all $n>n_{0}$ and this fact is represented by $\lim _{n \rightarrow \infty} x_{n}=x$ or $x_{n} \rightarrow x$ as $n \rightarrow \infty$;

(b) the sequence $\left\{x_{n}\right\}$ is said to be Cauchy sequence in $\left(X, r_{b}\right)$ if for every $\epsilon>0$ there exists $n_{0} \in \mathbb{N}$ such that $r_{b}\left(x_{n}, x_{n+p}\right)<\epsilon$ for all $n>n_{0}, p>0$ or equivalently, if $\lim _{n \rightarrow \infty} r_{b}\left(x_{n}, x_{n+p}\right)=0$ for all $p>0$;

(c) $\left(X, r_{b}\right)$ is said to be a complete rectangular $b$-metric space if every Cauchy sequence in $X$ converges to some $x \in X$.

Note that limit of sequence in a rectangular b-metric space is not necessarily unique and also every rectangular $b$-metric convergent sequence in a rectangular b-metric space is not necessarily rectangular b-metric-Cauchy ([11, Example 2.7]).

Definition 1.5 ([13]). An element $(x, y) \in X \times X$ is called a coupled fixed point of the mapping $F: X \times X \rightarrow$ $X$, if $F(x, y)=x, F(y, x)=y$.

Definition 1.6 ([17]). An element $(x, y) \in X \times X$ is called a coupled coincidence point of the mappings $F: X \times X \rightarrow X$ and $g: X \rightarrow X$, if $F(x, y)=g x, F(y, x)=g y$, and in this case, $(g x, g y)$ is called a coupled point of coincidence.

Definition 1.7 ([17]). An element $(x, y) \in X \times X$ is called a common coupled fixed point of mappings $F: X \times X \rightarrow X$ and $g: X \rightarrow X$, if $F(x, y)=g x=x, F(y, x)=g y=y$.

Definition 1.8 ([1]). Let $X$ be a nonempty set. A pair of mappings $F: X \times X \rightarrow X$ and $g: X \rightarrow X$ are called to be $w$-compatible, if $F(x, y)=g x$ and $F(y, x)=g y$, then we have $g F(x, y)=F(g x, g y)$. 


\section{Main results}

Theorem 2.1. Let $\mathrm{X}$ be a nonempty set and $\mathrm{r}_{\mathrm{b} 1}, \mathrm{r}_{\mathrm{b} 2}$ are two rectangular $\mathrm{b}$-metrics in $\mathrm{X}$ such that $\mathrm{r}_{\mathrm{b} 2}(\mathrm{x}, \mathrm{y}) \leqslant$ $\mathrm{r}_{\mathrm{b} 1}(\mathrm{x}, \mathrm{y})$, for all $\mathrm{x}, \mathrm{y} \in \mathrm{X}$. And the real number $\mathrm{s} \geqslant 1$ is the coefficient of rectangular $\mathrm{b}$-metric $\mathrm{d}_{1}$. Suppose that $\mathrm{F}: \mathrm{X} \times \mathrm{X} \rightarrow \mathrm{X}$ and $\mathrm{g}: \mathrm{X} \rightarrow \mathrm{X}$ are two mappings, and there exist $\mathrm{k}_{1}, \mathrm{k}_{2}$, and $\mathrm{k}_{3}$ in $[0,1)$ with $0 \leqslant \mathrm{k}_{1}+\mathrm{k}_{2}+\mathrm{k}_{3}<1$ and $0 \leqslant s k_{3}<1$ such that the condition

$$
\begin{aligned}
r_{\mathrm{b} 1}(F(x, y), F(u, v))+r_{b 1}(F(y, x), F(v, u)) \leqslant & k_{1}\left[r_{b 2}(g x, g u)+r_{b 2}(g y, g v)\right] \\
& +k_{2}\left[r_{b 2}(g x, F(x, y))+r_{b 2}(g y, F(y, x))\right] \\
& +k_{3}\left[r_{b 2}(g u, F(u, v))+r_{b 2}(g v, F(v, u))\right]
\end{aligned}
$$

holds for all $(x, y),(u, v) \in X \times X$. If $F(X \times X) \subset g(X)$ and $g(X)$ is $r_{b 1}$-complete, then $F$ and $g$ have a coupled coincidence point $(x, y) \in X \times X$, satisfying that $g x=F(x, y)=g y=F(y, x)$. Moreover, if $F$ and $g$ are $w$ compatible, then $\mathrm{F}$ and $\mathrm{g}$ have a unique common coupled fixed point of the form $(\mathrm{u}, \mathrm{u})$, which satisfies that $\mathrm{u}=$ $\mathrm{gu}=\mathrm{F}(\mathrm{u}, \mathrm{u})$.

Proof. Let $\left(x_{0}, y_{0}\right) \in X \times X$, by making the use of $F(X \times X) \subset g(X)$, then there exist $x_{1}, y_{1} \in X$ such that $g x_{1}=F\left(x_{0}, y_{0}\right), g y_{1}=F\left(y_{0}, x_{0}\right)$, and $g z_{1}=F\left(z_{0}, x_{0}\right)$. By similar arguments as above, we can show that there exist $x_{2}, y_{2} \in X$ such that $g x_{2}=T\left(x_{1}, y_{1}\right)$ and $g y_{2}=T\left(y_{1}, x_{1}\right), \ldots$. Repeating the above procedure, we can construct two sequences $\left\{x_{n}\right\}$ and $\left\{y_{n}\right\}$ such that

$$
g x_{n+1}=F\left(x_{n}, y_{n}\right), \quad g y_{n+1}=F\left(y_{n}, x_{n}\right), \quad \forall n \geqslant 0 .
$$

By taking $(x, y)=\left(x_{n}, y_{n}\right)$ and $(u, v)=\left(x_{n+1}, y_{n+1}\right)$ in $(2.1)$, we obtain

$$
\begin{aligned}
& r_{b 1}\left(g x_{n+1}, g x_{n+2}\right)+r_{b 1}\left(g y_{n+1}, g y_{n+2}\right) \\
&= r_{b 1}\left(F\left(x_{n}, y_{n}\right), F\left(x_{n+1}, y_{n+1}\right)\right)+r_{b 1}\left(F\left(y_{n}, x_{n}\right), F\left(y_{n+1}, x_{n+1}\right)\right) \\
& \leqslant k_{1}\left[r_{b 2}\left(g x_{n}, g x_{n+1}\right)+r_{b 2}\left(g y_{n}, g y_{n+1}\right)\right]+k_{2}\left[r_{b 2}\left(g x_{n}, F\left(x_{n}, y_{n}\right)\right)+r_{b 2}\left(g y_{n}, F\left(y_{n}, x_{n}\right)\right)\right] \\
&+k_{3}\left[r_{b 2}\left(g x_{n+1}, F\left(x_{n+1}, y_{n+1}\right)\right)+r_{b 2}\left(g y_{n+1}, F\left(y_{n+1}, x_{n+1}\right)\right)\right] \\
&= k_{1}\left[r_{b 2}\left(g x_{n}, g x_{n+1}\right)+r_{b 2}\left(g y_{n}, g y_{n+1}\right)\right]+k_{2}\left[r_{b 2}\left(g x_{n}, g x_{n+1}\right)+r_{b 2}\left(g y_{n}, g y_{n+1}\right)\right] \\
&+k_{3}\left[r_{b 2}\left(g x_{n+1}, g x_{n+2}\right)+r_{b 2}\left(g y_{n+1}, g y_{n+2}\right)\right] \\
& \leqslant k_{1}\left[r_{b 1}\left(g x_{n}, g x_{n+1}\right)+r_{b 1}\left(g y_{n}, g y_{n+1}\right)\right]+k_{2}\left[r_{b 1}\left(g x_{n}, g x_{n+1}\right)+r_{b 1}\left(g y_{n}, g y_{n+1}\right)\right] \\
&+k_{3}\left[r_{b 1}\left(g x_{n+1}, g x_{n+2}\right)+r_{b 1}\left(g y_{n+1}, g y_{n+2}\right)\right] .
\end{aligned}
$$

It follows from (2.2) that

$$
\begin{aligned}
r_{b 1}\left(g x_{n+1}, g x_{n+2}\right)+r_{b 1}\left(g y_{n+1}, g y_{n+2}\right) & \leqslant\left(\frac{k_{1}+k_{2}}{1-k_{3}}\right)\left[r_{b 1}\left(g x_{n}, g x_{n+1}\right)+r_{b 1}\left(g y_{n}, g y_{n+1}\right)\right] \\
& =k\left[r_{b 1}\left(g x_{n}, g x_{n+1}\right)+r_{b 1}\left(g y_{n}, g y_{n+1}\right)\right]
\end{aligned}
$$

where $k=\frac{k_{1}+k_{2}}{1-k_{3}}$, by the condition $0 \leqslant k_{1}+k_{2}+k_{3}<1$, then we have $0 \leqslant k<1$. By taking $\delta_{n}=$ $r_{b 1}\left(g x_{n}, g x_{n+1}\right)+r_{b 1}\left(g y_{n}, g y_{n+1}\right)$ and repetition of the above inequality (2.3) $n+1$ times, we deduce that

$$
\delta_{n+1} \leqslant k \delta_{n} \leqslant k^{2} \delta_{n-1} \leqslant \cdots \leqslant k^{n+1} \delta_{0} .
$$

As $(x, y)=\left(x_{n}, y_{n}\right)$ and $(u, v)=\left(x_{n+2}, y_{n+2}\right)$ in (2.1), also with (2.4), we get

$$
\begin{aligned}
r_{b 1}\left(g x_{n+1}, g x_{n+3}\right)+r_{b 1}\left(g y_{n+1}, g y_{n+3}\right) & \\
= & r_{b 1}\left(F\left(x_{n}, y_{n}\right), F\left(x_{n+2}, y_{n+2}\right)\right)+r_{b 1}\left(F\left(y_{n}, x_{n}\right), F\left(y_{n+2}, x_{n+2}\right)\right) \\
\leqslant & k_{1}\left[r_{b 2}\left(g x_{n}, g x_{n+2}\right)+r_{b 2}\left(g y_{n}, g y_{n+2}\right)\right]+k_{2}\left[r_{b 2}\left(g x_{n}, F\left(x_{n}, y_{n}\right)\right)+r_{b 2}\left(g y_{n}, F\left(y_{n}, x_{n}\right)\right)\right] \\
& +k_{3}\left[r_{b 2}\left(g x_{n+2}, F\left(x_{n+2}, y_{n+2}\right)\right)+r_{b 2}\left(g y_{n+2}, F\left(y_{n+2}, x_{n+2}\right)\right)\right]
\end{aligned}
$$




$$
\begin{aligned}
= & k_{1}\left[r_{b 2}\left(g x_{n}, g x_{n+2}\right)+r_{b 2}\left(g y_{n}, g y_{n+2}\right)\right]+k_{2}\left[r_{b 2}\left(g x_{n}, g x_{n+1}\right)+r_{b 2}\left(g y_{n}, g y_{n+1}\right)\right] \\
& +k_{3}\left[r_{b 2}\left(g x_{n+2}, g x_{n+3}\right)+r_{b 2}\left(g y_{n+2}, g y_{n+3}\right)\right] \\
\leqslant & k_{1}\left[r_{b 2}\left(g x_{n}, g x_{n+2}\right)+r_{b 2}\left(g y_{n}, g y_{n+2}\right)\right]+k_{2}\left[r_{b 2}\left(g x_{n}, g x_{n+1}\right)+r_{b 2}\left(g y_{n}, g y_{n+1}\right)\right] \\
& +k_{3} k^{2}\left[r_{b 2}\left(g x_{n}, g x_{n+1}\right)+r_{b 2}\left(g y_{n}, g y_{n+1}\right)\right] \\
= & k_{1}\left[r_{b 2}\left(g x_{n}, g x_{n+2}\right)+r_{b 2}\left(g y_{n}, g y_{n+2}\right)\right]+\left(k_{2}+k_{3} k^{2}\right)\left[r_{b 2}\left(g x_{n}, g x_{n+1}\right)+r_{b 2}\left(g y_{n}, g y_{n+1}\right)\right] \\
\leqslant & k_{1}\left[r_{b 1}\left(g x_{n}, g x_{n+2}\right)+r_{b 1}\left(g y_{n}, g y_{n+2}\right)\right]+\left(k_{2}+k_{3} k^{2}\right)\left[r_{b 1}\left(g x_{n}, g x_{n+1}\right)+r_{b 1}\left(g y_{n}, g y_{n+1}\right)\right] .
\end{aligned}
$$

By taking

$$
\delta_{n}^{*}=r_{b 1}\left(g x_{n}, g x_{n+2}\right)+r_{b 1}\left(g y_{n}, g y_{n+2}\right)
$$

and from $k=\frac{k_{1}+k_{2}}{1-k_{3}} \in[0,1)$, we have

$$
k_{1}+k_{2}+k_{3} k^{2} \leqslant k_{1}+k_{2}+k_{3} k=k_{1}+k_{2}+k_{3} \frac{k_{1}+k_{2}}{1-k_{3}}=\frac{k_{1}+k_{2}}{1-k_{3}}=k .
$$

Consequently, by the use of (2.4) and (2.5), we have

$$
\delta_{n+1}^{*} \leqslant k_{1} \delta_{n}^{*}+\left(k_{2}+k_{3} k^{2}\right) \delta_{n} \leqslant\left(k_{1}+k_{2}+k_{3} k^{2}\right) \max \left\{\delta_{n}, \delta_{n}^{*}\right\} \leqslant k \max \left\{\delta_{n}, \delta_{n}^{*}\right\} .
$$

It follows from (2.4) and (2.6) that

$$
\begin{aligned}
\delta_{n+1}^{*} \leqslant k \max \left\{\delta_{n}, \delta_{n}^{*}\right\} & \leqslant k \max \left\{k \delta_{n-1}, k \max \left\{\delta_{n-1}, \delta_{n-1}^{*}\right\}\right\} \\
& =k^{2} \max \left\{\delta_{n-1}, \delta_{n-1}^{*}\right\} \leqslant k^{3} \max \left\{\delta_{n-2}, \delta_{n-2}^{*}\right\} \leqslant \cdots \leqslant k^{n+1} \max \left\{\delta_{0}, \delta_{0}^{*}\right\} .
\end{aligned}
$$

Next, we show that $\left\{g x_{n}\right\}$ and $\left\{g y_{n}\right\}$ are Cauchy sequences in $g(X)$. For this, we consider $r_{b 1}\left(x_{n}, x_{n+p}\right)$ two cases.

Case 1. $p$ is an odd number, assume that $p=2 m+1$, then using (Rb3) we obtain

$$
\begin{aligned}
r_{b 1}\left(g x_{n}, g x_{n+p}\right)= & r_{b 1}\left(g x_{n}, g x_{n+2 m+1}\right) \\
\leqslant & s\left[r_{b 1}\left(g x_{n}, g x_{n+1}\right)+r_{b 1}\left(g x_{n+1}, g x_{n+2}\right)+r_{b 1}\left(g x_{n+2}, g x_{n+2 m+1}\right)\right] \\
\leqslant & s\left[r_{b 1}\left(g x_{n}, g x_{n+1}\right)+r_{b 1}\left(g x_{n+1}, g x_{n+2}\right)\right] \\
& +s^{2}\left[r_{b 1}\left(g x_{n+2}, g x_{n+3}\right)+r_{b 1}\left(g x_{n+3}, g x_{n+4}\right)+r_{b 1}\left(g x_{n+4}, g x_{n+2 m+1}\right)\right] \\
\leqslant & s\left[r_{b 1}\left(g x_{n}, g x_{n+1}\right)+r_{b 1}\left(g x_{n+1}, g x_{n+2}\right)\right]+s^{2}\left[r_{b 1}\left(g x_{n+2,} g x_{n+3}\right)+r_{b 1}\left(g x_{n+3}, g x_{n+4}\right)\right] \\
& +s^{3}\left[r_{b 1}\left(g x_{n+4}, g x_{n+5}\right)+r_{b 1}\left(g x_{n+5}, g x_{n+6}\right)+r_{b 1}\left(g x_{n+6}, g x_{n+2 m+1}\right)\right] \\
\leqslant & \cdots \\
\leqslant & s\left[r_{b 1}\left(g x_{n}, g x_{n+1}\right)+r_{b 1}\left(g x_{n+1}, g x_{n+2}\right)\right]+s^{2}\left[r_{b 1}\left(g x_{n+2,} g x_{n+3}\right)+r_{b 1}\left(g x_{n+3}, g x_{n+4}\right)\right] \\
& +s^{3}\left[r_{b 1}\left(g x_{n+4}, g x_{n+5}\right)+r_{b 1}\left(g x_{n+5}, g x_{n+6}\right)\right]+\cdots \\
& +s^{m}\left[r_{b 1}\left(g x_{n+2 m-2} g x_{n+2 m-1}\right)+r_{b 1}\left(g x_{n+2 m-1}, g x_{n+2 m}\right)+r_{b 1}\left(g x_{n+2 m}, g x_{n+2 m+1}\right)\right] .
\end{aligned}
$$

That is

$$
\begin{aligned}
& r_{b 1}\left(g x_{n}, g x_{n+p}\right) \\
&= r_{b 1}\left(g x_{n}, g x_{n+2 m+1}\right) \\
& \leqslant s\left[r_{b 1}\left(g x_{n}, g x_{n+1}\right)+r_{b 1}\left(g x_{n+1}, g x_{n+2}\right)\right]+s^{2}\left[r_{b 1}\left(g x_{n+2}, g x_{n+3}\right)+r_{b 1}\left(g x_{n+3}, g x_{n+4}\right)\right] \\
&+s^{3}\left[r_{b 1}\left(g x_{n+4}, g x_{n+5}\right)+r_{b 1}\left(g x_{n+5}, g x_{n+6}\right)\right]+\cdots \\
&+s^{m}\left[r_{b 1}\left(g x_{n+2 m-2}, g x_{n+2 m-1}\right)+r_{b 1}\left(g x_{n+2 m-1}, g x_{n+2 m}\right)\right]+s^{m} r_{b 1}\left(g x_{n+2 m}, g x_{n+2 m+1}\right) .
\end{aligned}
$$

We can similarly prove the following result 


$$
\begin{aligned}
& r_{b 1}\left(g y_{n}, g y_{n+p}\right) \\
&= r_{b 1}\left(g y_{n}, g y_{n+2 m+1}\right) \\
& \leqslant s\left[r_{b 1}\left(g y_{n}, g y_{n+1}\right)+r_{b 1}\left(g y_{n+1}, g y_{n+2}\right)\right]+s^{2}\left[r_{b 1}\left(g y_{n+2}, g y_{n+3}\right)+r_{b 1}\left(g y_{n+3}, g y_{n+4}\right)\right] \\
&+s^{3}\left[r_{b 1}\left(g y_{n+4}, g y_{n+5}\right)+r_{b 1}\left(g y_{n+5}, g y_{n+6}\right)\right]+\cdots \\
&+s^{m}\left[r_{b 1}\left(g y_{n+2 m-2}, g y_{n+2 m-1}\right)+r_{b 1}\left(g y_{n+2 m-1}, g y_{n+2 m}\right)\right]+s^{m} r_{b 1}\left(g y_{n+2 m}, g y_{n+2 m+1}\right) .
\end{aligned}
$$

Combining (2.4), (2.8), and (2.9), we have

$$
\begin{aligned}
& r_{b 1}\left(g x_{n}, g x_{n+p}\right)+r_{b 1}\left(g y_{n}, g y_{n+p}\right) \\
& =r_{b 1}\left(g x_{n}, g x_{n+2 m+1}\right)+r_{b 1}\left(g y_{n}, g y_{n+2 m+1}\right) \\
& \leqslant s\left(\delta_{n}+\delta_{n+1}\right)+s^{2}\left(\delta_{n+2}+\delta_{n+3}\right)+\cdots+s^{m}\left(\delta_{n+2 m-2}+\delta_{n+2 m-1}\right)+s^{m} \delta_{n+2 m} \\
& \leqslant s\left(k^{n}+k^{n+1}\right) \delta_{0}+s^{2}\left(k^{n+2}+k^{n+3}\right) \delta_{0}+\cdots+s^{m}\left(k^{n+2 m-2}+k^{n+2 m-1}\right) \delta_{0}+s^{m} k^{n+2 m} \delta_{0} \\
& =s k^{n}(1+k)\left[1+s k^{2}+\left(s k^{2}\right)^{2}+\cdots+\left(s k^{2}\right)^{m-1}\right] \delta_{0}+s^{m} k^{n+2 m} \delta_{0} \\
& = \begin{cases}{\left[s k^{n}(1+k) \cdot m+s^{m} k^{n+2 m}\right] \delta_{0},} & s k^{2}=1, \\
\left(s k^{n}(1+k) \cdot \frac{1-\left(s k^{2}\right)^{m}}{1-s k^{2}}+s^{m} k^{n+2 m}\right) \delta_{0}, & s k^{2} \neq 1,\end{cases} \\
& \leqslant \begin{cases}{\left[s k^{n}(1+k) \cdot m+s^{m} k^{n+2 m}\right] \delta_{0},} & s k^{2}=1, \\
\left(\frac{s k^{n}(1+k)}{1-s k^{2}}+s^{m} k^{n+2 m}\right) \delta_{0}, & s k^{2} \neq 1 .\end{cases}
\end{aligned}
$$

Case 2. $p$ is an even number, assume that $p=2 m$, then using ( $R b 3)$ we obtain

$$
\begin{aligned}
& r_{b 1}\left(g x_{n}, g x_{n+p}\right) \\
&= r_{b 1}\left(g x_{n}, g x_{n+2 m}\right) \\
& \leqslant s\left[r_{b 1}\left(g x_{n}, g x_{n+1}\right)+r_{b 1}\left(g x_{n+1}, g x_{n+2}\right)+r_{b 1}\left(g x_{n+2}, g x_{n+2 m}\right)\right] \\
& \leqslant s\left[r_{b 1}\left(g x_{n}, g x_{n+1}\right)+r_{b 1}\left(g x_{n+1}, g x_{n+2}\right)\right] \\
&+s^{2}\left[r_{b 1}\left(g x_{n+2}, g x_{n+3}\right)+r_{b 1}\left(g x_{n+3}, g x_{n+4}\right)+r_{b 1}\left(g x_{n+4}, g x_{n+2 m}\right)\right] \\
& \leqslant \cdots \\
& \leqslant s\left[r_{b 1}\left(g x_{n}, g x_{n+1}\right)+r_{b 1}\left(g x_{n+1}, g x_{n+2}\right)\right]+s^{2}\left[r_{b 1}\left(g x_{n+2}, g x_{n+3}\right)+r_{b 1}\left(g x_{n+3}, g x_{n+4}\right)\right] \\
&+\cdots+s^{m-1}\left[r_{b 1}\left(g x_{n+2 m-4}, g x_{n+2 m-3}\right)+r_{b 1}\left(g x_{n+2 m-3}, g x_{n+2 m-2}\right)\right] \\
&+s^{m-1} r_{b 1}\left(g x_{n+2 m-2}, g x_{n+2 m}\right) .
\end{aligned}
$$

By similar arguments as above,

$$
\begin{aligned}
& r_{b 1}\left(g y_{n}, g y_{n+p}\right) \\
&= r_{b 1}\left(g y_{n}, g y_{n+2 m}\right) \\
& \leqslant s\left[r_{b 1}\left(g y_{n}, g y_{n+1}\right)+r_{b 1}\left(g y_{n+1}, g y_{n+2}\right)\right]+s^{2}\left[r_{b 1}\left(g y_{n+2}, g y_{n+3}\right)+r_{b 1}\left(g y_{n+3}, g y_{n+4}\right)\right] \\
&+\cdots+s^{m-1}\left[r_{b 1}\left(g y_{n+2 m-4}, g y_{n+2 m-3}\right)+r_{b 1}\left(g y_{n+2 m-3}, g x_{n+2 m-2}\right)\right] \\
&+s^{m-1}\left[r_{b 1}\left(g y_{n+2 m-2}, g y_{n+2 m}\right)\right] .
\end{aligned}
$$

Combining (2.4), (2.7), (2.11) and (2.12), we have

$$
\begin{aligned}
& r_{b 1}\left(g x_{n}, g x_{n+p}\right)+r_{b 1}\left(g y_{n}, g y_{n+p}\right) \\
& \quad=r_{b 1}\left(g x_{n}, g x_{n+2 m}\right)+r_{b 1}\left(g y_{n}, g y_{n+2 m}\right)
\end{aligned}
$$




$$
\begin{aligned}
\leqslant & s\left(\delta_{n}+\delta_{n+1}\right)+s^{2}\left(\delta_{n+2}+\delta_{n+3}\right)+\cdots+s^{m-1}\left(\delta_{n+2 m-4}+\delta_{n+2 m-3}\right)+s^{m-1} \delta_{n+2 m-2}^{*} \\
\leqslant & s\left(k^{n}+k^{n+1}\right) \delta_{0}+s^{2}\left(k^{n+2}+k^{n+3}\right) \delta_{0} \\
& +\cdots+s^{m-1}\left(k^{n+2 m-4}+k^{n+2 m-3}\right) \delta_{0}+s^{m-1} k^{n+2 m-2} \max \left\{\delta_{0}, d_{0}^{*}\right\} \\
= & s k^{n}(1+k)\left[1+s k^{2}+\left(s k^{2}\right)^{2}+\cdots+\left(s k^{2}\right)^{m-2}\right] \delta_{0}+s^{m-1} k^{n+2 m-2} \max \left\{\delta_{0}, \delta_{0}^{*}\right\} \\
= & \left\{\begin{array}{lc}
s k^{n}(1+k)(m-1) \delta_{0}+s^{m-1} k^{n+2 m-2} \max \left\{\delta_{0}, \delta_{0}^{*}\right\}, & s k^{2}=1, \\
s k^{n}(1+k) \cdot \frac{1-\left(s k^{2}\right)^{m-1}}{1-s k^{2}} \cdot \delta_{0}+s^{m-1} k^{n+2 m-2} \max \left\{d_{0}, d_{0}^{*}\right\}, & s k^{2} \neq 1 .
\end{array}\right. \\
\leqslant & \left\{\begin{array}{lc}
s k^{n}(1+k)(m-1) \delta_{0}+s^{m-1} k^{n+2 m-2} \max \left\{\delta_{0}, \delta_{0}^{*}\right\}, & s k^{2}=1, \\
\frac{s k^{n}(1+k)}{1-s k^{2}} \cdot \delta_{0}+s^{m-1} k^{n+2 m-2} \max \left\{\delta_{0}, \delta_{0}^{*}\right\}, & s k^{2} \neq 1 .
\end{array}\right.
\end{aligned}
$$

Since $k \in[0,1)$, so $k^{n} \rightarrow 0$ as $n \rightarrow \infty$. Taking limit as $n \rightarrow \infty$ in (2.10) and (2.13), we get

$$
\lim _{n \rightarrow \infty}\left[r_{b 1}\left(g x_{n}, g x_{n+p}\right)+r_{b 1}\left(g y_{n}, g y_{n+p}\right)\right]=0,
$$

which implies that $\left\{g x_{n}\right\}$ and $\left\{g y_{n}\right\}$ are Cauchy sequences in $g(X)$. Since $g(X)$ is complete, then there exist $x, y \in X$ such that

$$
\lim _{n \rightarrow \infty} g x_{n}=g x \text { and } \lim _{n \rightarrow \infty} g y_{n}=g y .
$$

It follows from (2.1) and (2.4) that

$$
\begin{aligned}
& r_{b 1}\left(g x_{n+1}, F(x, y)\right)+r_{b 1}\left(g y_{n+1}, F(y, x)\right) \\
&=r_{b 1}\left(F\left(x_{n}, y_{n}\right), F(x, y)\right)+r_{b 1}\left(F\left(y_{n}, x_{n}\right), F(y, x)\right) \\
& \leqslant k_{1}\left[r_{b 2}\left(g x_{n}, g x\right)+r_{b 2}\left(g y_{n}, g y\right)\right]+k_{2}\left[r_{b 2}\left(g x_{n}, F\left(x_{n}, y_{n}\right)\right)+r_{b 2}\left(g y_{n}, F\left(y_{n}, x_{n}\right)\right)\right] \\
&+k_{3}\left[r_{b 2}(g x, F(x, y))+r_{b 2}(g y, F(y, x))\right] \\
&= k_{1}\left[r_{b 2}\left(g x_{n}, g x\right)+r_{b 2}\left(g y_{n}, g y\right)\right]+k_{2}\left[r_{b 2}\left(g x_{n}, g x_{n+1}\right)+r_{b 2}\left(g y_{n}, g y_{n+1}\right)\right] \\
&+k_{3}\left[r_{b 2}(g x, F(x, y))+r_{b 2}(g y, F(y, x))\right] \\
& \leqslant k_{1}\left[r_{b 1}\left(g x_{n}, g x\right)+r_{b 1}\left(g y_{n}, g y\right)\right]+k_{2}\left[r_{b 1}\left(g x_{n}, g x_{n+1}\right)+r_{b 1}\left(g y_{n}, g y_{n+1}\right)\right] \\
&+k_{3}\left[r_{b 1}(g x, F(x, y))+r_{b 1}(g y, F(y, x))\right] \\
&= k_{1}\left[r_{b 1}\left(g x_{n}, g x\right)+r_{b 1}\left(g y_{n}, g y\right)\right]+k_{2} \delta_{n}+k_{3}\left[r_{b 1}(g x, F(x, y))+r_{b 1}(g y, F(y, x))\right] \\
& \leqslant k_{1}\left[r_{b 1}\left(g x_{n}, g x\right)+r_{b 1}\left(g y_{n}, g y\right)\right]+k_{2} k^{n} \delta_{0}+k_{3}\left[r_{b 1}(g x, F(x, y))+r_{b 1}(g y, F(y, x))\right] .
\end{aligned}
$$

Applying (Rb3), (2.14), and (2.4) we have

$$
\begin{aligned}
r_{b 1}(g x, F(x, y))+r_{b 1}(g y, F(y, x)) \leqslant & s\left[r_{b 1}\left(g x, g x_{n}\right)+r_{b 1}\left(g x_{n}, g x_{n+1}\right)+r_{b 1}\left(g x_{n+1}, F(x, y)\right)\right] \\
& +s\left[r_{b 1}\left(g y, g y_{n}\right)+r_{b 1}\left(g y_{n}, g y_{n+1}\right)+r_{b 1}\left(g y_{n+1}, F(y, x)\right)\right] \\
= & s\left[r_{b 1}\left(g x, g x_{n}\right)+r_{b 1}\left(g y, g y_{n}\right)\right]+s \delta_{n} \\
& +s\left[r_{b 1}\left(g x_{n+1}, F(x, y)\right)+r_{b 1}\left(g y_{n+1}, F(y, x)\right)\right] \\
\leqslant & s\left(1+k_{1}\right)\left[r_{b 1}\left(g x_{n}, g x\right)+r_{b 1}\left(g y_{n}, g y\right)\right]+s\left(1+k_{2}\right) k^{n} \delta_{0} \\
& +s k_{3}\left[r_{b 1}(g x, F(x, y))+r_{b 1}(g y, F(y, x))\right] .
\end{aligned}
$$

By taking $n \rightarrow \infty$ in the above inequality (2.15), we have

$$
r_{b 1}(g x, F(x, y))+r_{b 1}(g y, F(y, x)) \leqslant s k_{3}\left[r_{b 1}(g x, F(x, y))+r_{b 1}(g y, F(y, x))\right] .
$$

By condition $0 \leqslant s k_{3}<1$ and (2.16), we can easily obtain that

$$
r_{b 1}(g x, F(x, y))+r_{b 1}(g y, F(y, x))=0,
$$


which implies that

$$
g x=F(x, y) \text { and } g y=F(y, x) .
$$

Therefore, we conclude that $(x, y)$ is the coupled coincidence point of $F$ and $g$.

Next, we show the uniqueness of the triple point of coincidence of $F$ and $g$. Assume that $\left(x^{*}, y^{*}\right)$ is another tripled coincidence point of mappings $F$ and $g$. By (2.1), we derive

$$
\begin{aligned}
r_{b 1}\left(g x, g x^{*}\right)+r_{b 1}\left(g y, g y^{*}\right)= & r_{b 1}\left(F(x, y), F\left(x^{*}, y^{*}\right)\right)+r_{b 1}\left(F(y, x), F\left(y^{*}, x^{*}\right)\right) \\
\leqslant & k_{1}\left[r_{b 2}\left(g x, g x^{*}\right)+r_{b 2}\left(g y, g y^{*}\right)\right]+k_{2}\left[r_{b 2}(g x, F(x, y))\right. \\
& \left.+r_{b 2}(g y, F(y, x))\right]+k_{3}\left[r_{b 2}\left(g x^{*}, F\left(x^{*}, y^{*}\right)\right)+r_{b 2}\left(g y^{*}, F\left(y^{*}, x^{*}\right)\right)\right] \\
= & k_{1}\left[r_{b 2}\left(g x, g x^{*}\right)+r_{b 2}\left(g y, g y^{*}\right)\right] \\
\leqslant & k_{1}\left[r_{b 1}\left(g x, g x^{*}\right)+r_{b 1}\left(g y, g y^{*}\right)\right] .
\end{aligned}
$$

By virtue of $0 \leqslant k_{1} \leqslant k_{1}+k_{2}+k_{3}<1$ and (2.17), we deduce

$$
r_{b 1}\left(g x, g x^{*}\right)+r_{b 1}\left(g y, g y^{*}\right)=0 .
$$

This implies that $g x=g x^{*}$ and $g y=g y^{*}$. So that the couple point of coincidence of $F$ and $g$ is unique.

Next, we show that $g x=g y$. In fact, it follows from (2.1) that

$$
\begin{aligned}
r_{b 1}(g x, g y)+r_{b 1}(g y, g x)= & r_{b 1}(F(x, y), F(y, x))+r_{b 1}(F(y, x), F(x, y)) \\
\leqslant & k_{1}\left[r_{b 2}(g x, g y)+r_{b 2}(g y, g x)\right]+k_{2}\left[r_{b 2}(g x, F(x, y))+r_{b 2}(g y, F(y, x))\right] \\
& +k_{3}\left[r_{b 2}(g y, F(y, x))+r_{b 2}(g x, F(x, y))\right] \\
= & k_{1}\left[r_{b 2}(g x, g y)+r_{b 2}(g y, g x)\right] \\
\leqslant & k_{1}\left[r_{b 1}(g x, g y)+r_{b 1}(g y, g x)\right] .
\end{aligned}
$$

By making use of $0 \leqslant k_{1} \leqslant k_{1}+k_{2}+k_{3}<1$ and (2.17), we deduce

$$
r_{\mathrm{b} 1}(g x, g y)+r_{b 1}(g y, g x)=0 .
$$

This means that $g x=g y$.

Finally, if $F$ and $g$ are $w$-compatible, then we have $g(F(x, y))=F(g x, g y)$. Therefore, by taking $u=g x$, we have $u=g x=F(x, y)=g y=F(y, x)$, hence we have

$$
g u=g g x=g(F(x, y))=F(g x, g y)=F(u, u) .
$$

Consequently, $(g u, g u)$ is a coupled point of coincidence of $g$ and $F$, and by its uniqueness, we get $g u=g x$. Thus, we obtain $F(u, u)=g u=u$. Therefore, $(u, u)$ is the unique common coupled fixed point of $g$ and F. This completes the proof of Theorem 2.1.

Corollary 2.2. Let $\mathrm{r}_{\mathrm{b} 1}$ and $\mathrm{r}_{\mathrm{b} 2}$ be two rectangular b-metrics on $\mathrm{X}$ such that $\mathrm{r}_{\mathrm{b} 2}(\mathrm{x}, \mathrm{y}) \leqslant \mathrm{r}_{\mathrm{b} 1}(\mathrm{x}, \mathrm{y})$ for all $\mathrm{x}, \mathrm{y} \in \mathrm{X}$, $\left(\mathrm{X}, \mathrm{r}_{\mathrm{b} 1}\right)$ with coefficient $\mathrm{s} \geqslant 1$, and $\mathrm{F}: \mathrm{X} \times \mathrm{X} \rightarrow \mathrm{X}$ and $\mathrm{g}: \mathrm{X} \rightarrow \mathrm{X}$ be two mappings. Suppose that there exist $\mathrm{k}_{1}, \mathrm{k}_{2}$, and $\mathrm{k}_{3}$ in $[0,1)$ with $0 \leqslant 2\left(\mathrm{k}_{1}+\mathrm{k}_{2}+\mathrm{k}_{3}\right)<1$ and $0 \leqslant 2 \mathrm{sk}_{3}<1$ such that the condition

$$
\begin{aligned}
r_{b 1}(F(x, y), F(u, v)) \leqslant & k_{1}\left[r_{b 2}(g x, g u)+r_{b 2}(g y, g v)\right]+k_{2}\left[r_{b 2}(g x, F(x, y))+r_{b 2}(g y, F(y, x))\right] \\
& +k_{3}\left[r_{b 2}(g u, F(u, v))+r_{b 2}(g v, F(v, u))\right]
\end{aligned}
$$

holds for all $(x, y),(u, v) \in X \times X$. If $F(X \times X) \subset g(X)$ and $g(X)$ is $r_{b 1}$-complete, then $F$ and $g$ have a coupled coincidence point $(x, y) \in X \times X$, which satisfies that $g x=F(x, y)=g y=F(y, x)$. Moreover, if $F$ and $g$ are $w$-compatible, then $\mathrm{F}$ and $\mathrm{g}$ have a unique common coupled fixed point of the form $(\mathrm{u}, \mathrm{u})$, which satisfies that $\mathrm{u}=\mathrm{gu}=\mathrm{F}(\mathrm{u}, \mathrm{u})$. 
Proof. It follows from (2.18) that

$$
\begin{aligned}
r_{b 1}(F(x, y), F(u, v)) \leqslant & k_{1}\left[r_{b 2}(g x, g u)+r_{b 2}(g y, g v)\right]+k_{2}\left[r_{b 2}(g x, F(x, y))+r_{b 2}(g y, F(y, x))\right] \\
& +k_{3}\left[r_{b 2}(g u, F(u, v))+r_{b 2}(g v, F(v, u))\right]
\end{aligned}
$$

and

$$
\begin{aligned}
r_{b 1}(F(y, x), F(v, u)) \leqslant & k_{1}\left[r_{b 2}(g y, g v)+r_{b 2}(g x, g u)\right]+k_{2}\left[r_{b 2}(g y, F(y, x))+r_{b 2}(g x, F(x, y))\right] \\
& +k_{3}\left[r_{b 2}(g v, F(v, u))+r_{b 2}(g u, F(u, v))\right] .
\end{aligned}
$$

Combining (2.19) and (2.20), we have

$$
\begin{aligned}
r_{\mathrm{b} 1}(F(x, y), F(u, v))+r_{b 1}(F(y, x), F(v, u)) \leqslant & 2 k_{1}\left[r_{b 2}(g x, g u)+r_{b 2}(g y, g v)\right]+2 k_{2}\left[r_{b 2}(g x, F(x, y))\right. \\
& \left.+r_{b 2}(g y, F(y, z))\right]+2 k_{3}\left[r_{b 2}(g u, F(u, v))+r_{b 2}(g v, F(v, u))\right] .
\end{aligned}
$$

By Theorem 2.1, we can get the conclusion.

In Theorem 2.1, if we take $r_{b 1}(x, y)=r_{b 2}(x, y)=r_{b}(x, y)$ for all $x, y \in X$, then we get the following corollary.

Corollary 2.3. Let $\left(\mathrm{X}, \mathrm{r}_{\mathrm{b}}\right)$ be a $\mathrm{r}_{\mathrm{b}}$-complete rectangular b-metric space with coefficient $\mathrm{s} \geqslant 1, \mathrm{~F}: \mathrm{X} \times \mathrm{X} \rightarrow \mathrm{X}$ and $\mathrm{g}: \mathrm{X} \rightarrow \mathrm{X}$ be two mappings. Suppose that there exist $\mathrm{k}_{1}, \mathrm{k}_{2}$, and $\mathrm{k}_{3}$ in $[0,1)$ with $0 \leqslant \mathrm{k}_{1}+\mathrm{k}_{2}+\mathrm{k}_{3}<1$ and $0 \leqslant s k_{3}<1$ such that the condition

$$
\begin{aligned}
& r_{b}(F(x, y), F(u, v))+r_{b}(F(y, x), F(v, u)) \\
& \quad \leqslant k_{1}\left[r_{b}(g x, g u)+r_{b}(g y, g v)\right]+k_{2}\left[r_{b}(g x, F(x, y))+r_{b}(g y, F(y, x))\right]+k_{3}\left[r_{b}(g u, F(u, v))+r_{b}(g v, F(v, u))\right]
\end{aligned}
$$

holds for all $(x, y),(u, v) \in X \times X$. If $F(X \times X) \subset g(X)$, then $F$ and $g$ have a coupled coincidence point $(x, y) \in$ $\mathrm{X} \times \mathrm{X}$, satisfying that $\mathrm{gx}=\mathrm{F}(\mathrm{x}, \mathrm{y})=\mathrm{gy}=\mathrm{F}(\mathrm{y}, \mathrm{x})$. Moreover, if $\mathrm{F}$ and $\mathrm{g}$ are $\mathrm{w}$-compatible, then $\mathrm{F}$ and $\mathrm{g}$ have a unique common coupled fixed point of the form $(\mathfrak{u}, \mathfrak{u})$, which satisfies that $\mathfrak{u}=g \mathfrak{u}=\mathrm{F}(\mathrm{u}, \mathfrak{u})$.

Corollary 2.4. Let $\mathrm{r}_{\mathrm{b} 1}$ and $\mathrm{r}_{\mathrm{b} 2}$ be two rectangular $\mathrm{b}$-metrics on $\mathrm{X}$ such that $\mathrm{r}_{\mathrm{b} 2}(\mathrm{x}, \mathrm{y}) \leqslant \mathrm{r}_{\mathrm{b} 1}(\mathrm{x}, \mathrm{y})$ for all $\mathrm{x}, \mathrm{y} \in \mathrm{X}$, $\left(\mathrm{X}, \mathrm{r}_{\mathrm{b} 1}\right)$ with coefficient $\mathrm{s} \geqslant 1$, and $\mathrm{F}: \mathrm{X} \times \mathrm{X} \rightarrow \mathrm{X}$ and $\mathrm{g}: \mathrm{X} \rightarrow \mathrm{X}$ be two mappings. Suppose that there exist $a_{i} \in[0,1)(i=1,2,3, \cdots, 6)$ with $0 \leqslant a_{1}+a_{2}+a_{3}+\cdots+a_{6}<1$ and $0 \leqslant s\left(a_{5}+a_{6}\right)<1$ such that the condition

$$
\begin{aligned}
r_{b 1}(F(x, y), F(u, v)) \leqslant & a_{1} r_{b 2}(g x, g u)+a_{2} r_{b 2}(g y, g v)+a_{3} r_{b 2}(g x, F(x, y))+a_{4} r_{b 2}(g y, F(y, x)) \\
& +a_{5} r_{b 2}(g u, F(u, v))+a_{6} r_{b 2}(g v, F(v, u))
\end{aligned}
$$

holds for all $(\mathrm{x}, \mathrm{y}),(\mathrm{u}, v) \in \mathrm{X} \times \mathrm{X}$. If $\mathrm{F}(\mathrm{X} \times \mathrm{X}) \subset \mathrm{g}(\mathrm{X})$ and $\mathrm{g}(\mathrm{X})$ is $\mathrm{r}_{\mathrm{b} 1}$-complete, then $\mathrm{F}$ and $\mathrm{g}$ have a coupled coincidence point $(\mathrm{x}, \mathrm{y}) \in \mathrm{X} \times \mathrm{X}$, which satisfies that $\mathrm{gx}=\mathrm{F}(\mathrm{x}, \mathrm{y})=\mathrm{gy}=\mathrm{F}(\mathrm{y}, \mathrm{x})$. Moreover, if $\mathrm{F}$ and $\mathrm{g}$ are $w$-compatible, then $\mathrm{F}$ and $\mathrm{g}$ have a unique common coupled fixed point of the form $(\mathrm{u}, \mathrm{u})$, which satisfies that $\mathrm{u}=\mathrm{gu}=\mathrm{F}(\mathrm{u}, \mathrm{u})$.

Proof. Given $(x, y),(u, v) \in X \times X$, it follows from (2.21) that

$$
\begin{aligned}
r_{b 1}(F(x, y), F(u, v)) \leqslant & a_{1} r_{b 2}(g x, g u)+a_{2} r_{b 2}(g y, g v)+a_{3} r_{b 2}(g x, F(x, y))+a_{4} r_{b 2}(g y, F(y, x)) \\
& +a_{5} r_{b 2}(g u, F(u, v))+a_{6} r_{b 2}(g v, F(v, u))
\end{aligned}
$$

and

$$
\begin{aligned}
r_{b 1}(F(y, x), F(v, u)) \leqslant & a_{1} r_{b 2}(g y, g v)+a_{2} r_{b 2}(g x, g u)+a_{3} r_{b 2}(g y, F(y, x))+a_{4} r_{b 2}(g x, F(x, y)) \\
& +a_{5} r_{b 2}(g v, F(v, u))+a_{6} r_{b 2}(g u, F(u, v)) .
\end{aligned}
$$


Combining (2.22) and (2.23), we have

$$
\begin{aligned}
r_{b 1}(F(x, y), F(u, v))+r_{b 1}(F(y, x), F(v, u)) \leqslant & \left(a_{1}+a_{2}\right)\left[r_{b 2}(g x, g u)+r_{b 2}(g y, g v)\right] \\
& +\left(a_{3}+a_{4}\right)\left[r_{b 2}(g x, F(x, y))+r_{b 2}(g y, F(y, x))\right] \\
& +\left(a_{5}+a_{6}\right)\left[r_{b 2}(g u, F(u, v))+r_{b 2}(g v, F(v, u))\right] .
\end{aligned}
$$

Therefore, the result follows from Theorem 2.1.

Remark 2.5. If we take $r_{b 1}(x, y)=r_{b 2}(x, y)=r_{b}(x, y)$ for all $x, y \in X$, where $r_{b}$ is a rectangular $b$-metric on $X$, then Corollary 2.4 will be reduced to a new result.

The following corollary can be obtained from Theorem 2.1 immediately.

Corollary 2.6. Let $\mathrm{r}_{\mathrm{b} 1}$ and $\mathrm{r}_{\mathrm{b} 2}$ be two rectangular b-metrics on $\mathrm{X}$ such that $\mathrm{r}_{\mathrm{b} 2}(\mathrm{x}, \mathrm{y}) \leqslant \mathrm{r}_{\mathrm{b} 1}(\mathrm{x}, \mathrm{y})$ for all $\mathrm{x}, \mathrm{y} \in \mathrm{X}$, and $\mathrm{F}: \mathrm{X} \times \mathrm{X} \rightarrow \mathrm{X}$ and $\mathrm{g}: \mathrm{X} \rightarrow \mathrm{X}$ be two mappings. Suppose that there exists $\mathrm{k} \in[0,1)$ such that the condition

$$
r_{b 1}(F(x, y), F(u, v))+r_{b 1}(F(y, x), F(v, u)) \leqslant k\left[r_{b 2}(g x, g u)+r_{b 2}(g y, g v)\right]
$$

holds for all $(x, y),(u, v) \in X \times X$. If $F(X \times X) \subset g(X)$ and $g(X)$ is $r_{b 1}$-complete, then $F$ and $g$ have a coupled coincidence point $(\mathrm{x}, \mathrm{y}) \in \mathrm{X} \times \mathrm{X}$, which satisfies that $\mathrm{gx}=\mathrm{F}(\mathrm{x}, \mathrm{y})=\mathrm{gy}=\mathrm{F}(\mathrm{y}, \mathrm{x})$. Moreover, if $\mathrm{F}$ and $\mathrm{g}$ are $w$-compatible, then $\mathrm{F}$ and $\mathrm{g}$ have a unique common coupled fixed point of the form $(\mathrm{u}, \mathrm{u})$, which satisfies that $\mathrm{u}=\mathrm{gu}=\mathrm{F}(\mathrm{u}, \mathrm{u})$.

Corollary 2.7. Let $\mathrm{r}_{\mathrm{b} 1}$ and $\mathrm{r}_{\mathrm{b} 2}$ be two rectangular b-metrics on $\mathrm{X}$ such that $\mathrm{r}_{\mathrm{b} 2}(\mathrm{x}, \mathrm{y}) \leqslant \mathrm{r}_{\mathrm{b} 1}(\mathrm{x}, \mathrm{y})$ for all $\mathrm{x}, \mathrm{y} \in \mathrm{X}$, and $\mathrm{F}: \mathrm{X} \times \mathrm{X} \rightarrow \mathrm{X}$ and $\mathrm{g}: \mathrm{X} \rightarrow \mathrm{X}$ be two mappings. Suppose that there exists $\mathrm{k} \in[0,1)$ such that the condition

$$
r_{b 1}(F(x, y), F(u, v))+r_{b 1}(F(y, x), F(v, u)) \leqslant k\left[r_{b 2}(g x, F(x, y))+r_{b 2}(g y, F(y, x))\right]
$$

holds for all $(x, y),(u, v) \in X \times X$. If $F(X \times X) \subset g(X)$ and $g(X)$ is $r_{b 1}$-complete, then $F$ and $g$ have a coupled coincidence point $(x, y) \in X \times X$, which satisfies that $g x=F(x, y)=g y=F(y, x)$. Moreover, if $F$ and $g$ are $w$-compatible, then $\mathrm{F}$ and $\mathrm{g}$ have a unique common coupled fixed point of the form $(\mathrm{u}, \mathrm{u})$, which satisfies that $\mathrm{u}=\mathrm{gu}=\mathrm{F}(\mathrm{u}, \mathrm{u})$.

Corollary 2.8. Let $\mathrm{r}_{\mathrm{b} 1}$ and $\mathrm{r}_{\mathrm{b} 2}$ be two rectangular $\mathrm{b}$-metrics on $\mathrm{X}$ such that $\mathrm{r}_{\mathrm{b} 2}(\mathrm{x}, \mathrm{y}) \leqslant \mathrm{r}_{\mathrm{b} 1}(\mathrm{x}, \mathrm{y})$ for all $\mathrm{x}, \mathrm{y} \in \mathrm{X}$, $\left(\mathrm{X}, \mathrm{r}_{\mathrm{b} 1}\right)$ with coefficient $\mathrm{s} \geqslant 1$, and $\mathrm{F}: \mathrm{X} \times \mathrm{X} \rightarrow \mathrm{X}$ and $\mathrm{g}: \mathrm{X} \rightarrow \mathrm{X}$ be two mappings. Suppose that there exists $k \in[0,1)$ with $0 \leqslant s k<1$ such that the condition

$$
r_{b 1}(F(x, y), F(u, v))+r_{b 1}(F(y, x), F(v, u)) \leqslant k\left[r_{b 2}(g u, F(u, v))+r_{b 2}(g v, F(v, u))\right]
$$

holds for all $(\mathrm{x}, \mathrm{y}),(\mathrm{u}, v) \in \mathrm{X} \times \mathrm{X}$. If $\mathrm{F}(\mathrm{X} \times \mathrm{X}) \subset \mathrm{g}(\mathrm{X})$ and $\mathrm{g}(\mathrm{X})$ is $\mathrm{r}_{\mathrm{b} 1}$-complete, then $\mathrm{F}$ and $\mathrm{g}$ have a coupled coincidence point $(x, y) \in X \times X$, which satisfies that $g x=F(x, y)=g y=F(y, x)$. Moreover, if $F$ and $g$ are $w$-compatible, then $\mathrm{F}$ and $\mathrm{g}$ have a unique common coupled fixed point of the form $(\mathrm{u}, \mathrm{u})$, which satisfies that $\mathrm{u}=\mathrm{gu}=\mathrm{F}(\mathrm{u}, \mathrm{u})$.

Let $\mathrm{g}=\mathrm{I}_{\mathrm{X}}$ (the identity mapping) in Theorem 2.1 and Corollaries 2.2-2.4, 2.6, 2.7. Then we have the following results.

Corollary 2.9. Let $\mathrm{r}_{\mathrm{b} 1}$ and $\mathrm{r}_{\mathrm{b} 2}$ be two rectangular b-metrics on $\mathrm{X}$ such that $\mathrm{r}_{\mathrm{b} 2}(\mathrm{x}, \mathrm{y}) \leqslant \mathrm{r}_{\mathrm{b} 1}(\mathrm{x}, \mathrm{y})$ for all $\mathrm{x}, \mathrm{y} \in \mathrm{X}$, $\left(\mathrm{X}, \mathrm{r}_{\mathrm{b} 1}\right)$ with coefficient $\mathrm{s} \geqslant 1$ and $\mathrm{F}: \mathrm{X} \times \mathrm{X} \rightarrow \mathrm{X}$ be a mapping. Suppose that there exist $\mathrm{k}_{1}, \mathrm{k}_{2}$, and $\mathrm{k}_{3}$ in $[0,1)$ with $0 \leqslant k_{1}+k_{2}+k_{3}<1$ and $0 \leqslant s k_{3}<1$ such that the condition

$$
\begin{aligned}
r_{b 1}(F(x, y), F(u, v))+r_{b 1}(F(y, x), F(v, u)) \leqslant & k_{1}\left[r_{b 2}(x, u)+r_{b 2}(y, v)\right]+k_{2}\left[r_{b 2}(x, F(x, y))\right. \\
& \left.+r_{b 2}(y, F(y, x))\right]+k_{3}\left[r_{b 2}(u, F(u, v))+r_{b 2}(v, F(v, u))\right]
\end{aligned}
$$

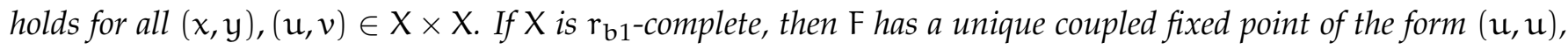
which satisfies that $\mathrm{u}=\mathrm{F}(\mathrm{u}, \mathrm{u})$. 
Corollary 2.10. Let $\mathrm{r}_{\mathrm{b} 1}$ and $\mathrm{r}_{\mathrm{b} 2}$ be two rectangular b-metrics on $\mathrm{X}$ such that $\mathrm{r}_{\mathrm{b} 2}(\mathrm{x}, \mathrm{y}) \leqslant \mathrm{r}_{\mathrm{b} 1}(\mathrm{x}, \mathrm{y})$ for all $\mathrm{x}, \mathrm{y} \in \mathrm{X}$, $\left(\mathrm{X}, \mathrm{r}_{\mathrm{b} 1}\right)$ with coefficient $\mathrm{s} \geqslant 1$ and $\mathrm{F}: \mathrm{X} \times \mathrm{X} \rightarrow \mathrm{X}$ be a mapping. Suppose that there exist $\mathrm{k}_{1}, \mathrm{k}_{2}$, and $\mathrm{k}_{3}$ in $[0,1)$ with $0 \leqslant 2\left(k_{1}+k_{2}+k_{3}\right)<1$ and $0 \leqslant 2 s_{3}<1$ such that the condition

$$
\begin{aligned}
r_{b 1}(F(x, y), F(u, v)) \leqslant & k_{1}\left[r_{b 2}(x, u)+r_{b 2}(y, v)\right]+k_{2}\left[r_{b 2}(x, F(x, y))+r_{b 2}(y, F(y, x))\right] \\
& +k_{3}\left[r_{b 2}(u, F(u, v))+r_{b 2}(v, F(v, u))\right]
\end{aligned}
$$

holds for all $(x, y),(u, v) \in X \times X$. If $g(X)$ is $r_{b 1}$-complete, then $F$ and $g$ have a coupled coincidence point $(x, y) \in$ $\mathrm{X} \times \mathrm{X}$, which satisfies that $\mathrm{gx}=\mathrm{F}(\mathrm{x}, \mathrm{y})=\mathrm{gy}=\mathrm{F}(\mathrm{y}, \mathrm{x})$. Moreover, if $\mathrm{F}$ and $\mathrm{g}$ are $\mathrm{w}$-compatible, then $\mathrm{F}$ and $\mathrm{g}$ have a unique common coupled fixed point of the form $(\mathrm{u}, \mathrm{u})$, which satisfies that $\mathrm{u}=\mathrm{gu}=\mathrm{F}(\mathrm{u}, \mathrm{u})$.

Corollary 2.11. Let $\left(\mathrm{X}, \mathrm{r}_{\mathrm{b}}\right)$ be a $\mathrm{r}_{\mathrm{b}}$-complete rectangular $\mathrm{b}$-metric space with coefficient $\mathrm{s} \geqslant 1$, and $\mathrm{F}: \mathrm{X} \times \mathrm{X} \rightarrow \mathrm{X}$ be a mapping. Suppose that there exist $\mathrm{k}_{1}, \mathrm{k}_{2}$, and $\mathrm{k}_{3}$ in $[0,1)$ with $0 \leqslant \mathrm{k}_{1}+\mathrm{k}_{2}+\mathrm{k}_{3}<1$ and $0 \leqslant s \mathrm{k}_{3}<1$ such that the condition

$$
\begin{aligned}
r_{b}(F(x, y), F(u, v))+r_{b}(F(y, x), F(v, u)) \leqslant & k_{1}\left[r_{b}(x, u)+r_{b}(y, v)\right]+k_{2}\left[r_{b}(x, F(x, y))+r_{b}(y, F(y, x))\right] \\
& +k_{3}\left[r_{b}(u, F(u, v))+r_{b}(v, F(v, u))\right]
\end{aligned}
$$

holds for all $(x, y),(u, v) \in X \times X$. Then $F$ has a unique coupled fixed point of the form $(u, u)$, which satisfies that $\mathrm{u}=\mathrm{gu}=\mathrm{F}(\mathrm{u}, \mathrm{u})$.

Corollary 2.12. Let $\mathrm{r}_{\mathrm{b} 1}$ and $\mathrm{r}_{\mathrm{b} 2}$ be two rectangular b-metrics on $\mathrm{X}$ such that $\mathrm{r}_{\mathrm{b} 2}(\mathrm{x}, \mathrm{y}) \leqslant \mathrm{r}_{\mathrm{b} 1}(\mathrm{x}, \mathrm{y})$ for all $\mathrm{x}, \mathrm{y} \in \mathrm{X}$, $\left(X, r_{b 1}\right)$ with coefficient $s \geqslant 1$ and $\mathrm{F}: X \times X \rightarrow X$ be a mapping. Suppose that there exist $\mathrm{a}_{i} \in[0,1)(i=$ $1,2,3, \cdots, 6)$ with $0 \leqslant a_{1}+a_{2}+a_{3}+\cdots+a_{6}<1$ and $0 \leqslant s\left(a_{5}+a_{6}\right)<1$ such that the condition

$$
\begin{aligned}
r_{b 1}(F(x, y), F(u, v)) \leqslant & a_{1} r_{b 2}(x, u)+a_{2} r_{b 2}(y, v)+a_{3} r_{b 2}(x, F(x, y))+a_{4} r_{b 2}(y, F(y, x)) \\
& +a_{5} r_{b 2}(u, F(u, v))+a_{6} r_{b 2}(v, F(v, u))
\end{aligned}
$$

holds for all $(x, y),(u, v) \in X \times X$. If $\left(X, r_{b 1}\right)$ is complete, then $F$ has a unique coupled fixed point of the form $(u, u)$, which satisfies that $\mathrm{u}=\mathrm{gu}=\mathrm{F}(\mathrm{u}, \mathrm{u})$.

Corollary 2.13. Let $\mathrm{r}_{\mathrm{b} 1}$ and $\mathrm{r}_{\mathrm{b} 2}$ be two rectangular b-metrics on $\mathrm{X}$ such that $\mathrm{r}_{\mathrm{b} 2}(\mathrm{x}, \mathrm{y}) \leqslant \mathrm{r}_{\mathrm{b} 1}(\mathrm{x}, \mathrm{y})$ for all $\mathrm{x}, \mathrm{y} \in \mathrm{X}$, and $\mathrm{F}: \mathrm{X} \times \mathrm{X} \rightarrow \mathrm{X}$ be a mapping. Suppose that there exists $\mathrm{k} \in[0,1)$ such that the condition

$$
r_{b 1}(F(x, y), F(u, v))+r_{b 1}(F(y, x), F(v, u)) \leqslant k\left[r_{b 2}(x, u)+r_{b 2}(y, v)\right]
$$

holds for all $(x, y),(u, v) \in X \times X$. If $\left(X, r_{b 1}\right)$ is complete, then $F$ has a unique coupled fixed point of the form $(u, u)$, which satisfies that $\mathrm{u}=\mathrm{gu}=\mathrm{F}(\mathrm{u}, \mathrm{u})$.

Corollary 2.14. Let $\mathrm{r}_{\mathrm{b} 1}$ and $\mathrm{r}_{\mathrm{b} 2}$ be two rectangular b-metrics on $\mathrm{X}$ such that $\mathrm{r}_{\mathrm{b} 2}(\mathrm{x}, \mathrm{y}) \leqslant \mathrm{r}_{\mathrm{b} 1}(\mathrm{x}, \mathrm{y})$ for all $\mathrm{x}, \mathrm{y} \in \mathrm{X}$, and $\mathrm{F}: \mathrm{X} \times \mathrm{X} \rightarrow \mathrm{X}$ be a mapping. Suppose that there exists $\mathrm{k} \in[0,1)$ such that the condition

$$
r_{b 1}(F(x, y), F(u, v))+r_{b 1}(F(y, x), F(v, u)) \leqslant k\left[r_{b 2}(x, F(x, y))+r_{b 2}(y, F(y, x))\right]
$$

holds for all $(x, y),(u, v) \in X \times X$. If $\left(X, r_{b 1}\right)$ is complete, then $F$ has a unique coupled fixed point of the form $(u, u)$, which satisfies that $\mathfrak{u}=\mathrm{gu}=\mathrm{F}(\mathrm{u}, \mathrm{u})$.

Corollary 2.15. Let $\mathrm{r}_{\mathrm{b} 1}$ and $\mathrm{r}_{\mathrm{b} 2}$ be two rectangular b-metrics on $\mathrm{X}$ such that $\mathrm{r}_{\mathrm{b} 2}(\mathrm{x}, \mathrm{y}) \leqslant \mathrm{r}_{\mathrm{b} 1}(\mathrm{x}, \mathrm{y})$ for all $\mathrm{x}, \mathrm{y} \in \mathrm{X}$, $\left(\mathrm{X}, \mathrm{r}_{\mathrm{b} 1}\right)$ with coefficient $\mathrm{s} \geqslant 1$, and $\mathrm{F}: \mathrm{X} \times \mathrm{X} \rightarrow \mathrm{X}$ and $\mathrm{g}: \mathrm{X} \rightarrow \mathrm{X}$ be two mappings. Suppose that there exists $k \in[0,1)$ with $0 \leqslant s k<1$ such that the condition

$$
r_{\mathrm{b} 1}(\mathrm{~F}(\mathrm{x}, \mathrm{y}), \mathrm{F}(\mathrm{u}, v))+\mathrm{r}_{\mathrm{b} 1}(\mathrm{~F}(\mathrm{y}, \mathrm{x}), \mathrm{F}(v, \mathrm{u})) \leqslant k\left[\mathrm{r}_{\mathrm{b} 2}(\mathrm{u}, \mathrm{F}(\mathrm{u}, v))+\mathrm{r}_{\mathrm{b} 2}(v, \mathrm{~F}(v, \mathrm{u}))\right]
$$

holds for all $(x, y),(u, v) \in X \times X$. If $\left(X, r_{b 1}\right)$ is complete, then $F$ has a unique coupled fixed point of the form $(u, u)$, which satisfies that $\mathrm{u}=\mathrm{gu}=\mathrm{F}(\mathrm{u}, \mathrm{u})$. 
It follows by taking coefficient $s=1$ (for the space $\mathrm{X}$ ) in Theorem 2.1 , then we have the following corollary.

Corollary 2.16. Let $X$ be a nonempty set and $r_{1}, r_{2}$ are two rectangular metrics in $X$ such that $r_{2}(x, y) \leqslant r_{1}(x, y)$, for all $\mathrm{x}, \mathrm{y} \in \mathrm{X}$. Suppose that $\mathrm{F}: \mathrm{X} \times \mathrm{X} \rightarrow \mathrm{X}$ and $\mathrm{g}: \mathrm{X} \rightarrow \mathrm{X}$ are two mappings, and there exist $\mathrm{k}_{1}, \mathrm{k}_{2}$, and $\mathrm{k}_{3}$ in $[0,1)$ with $0 \leqslant k_{1}+k_{2}+k_{3}<1$ such that the condition

$$
\begin{aligned}
& r_{1}(F(x, y), F(u, v))+r_{1}(F(y, x), F(v, u)) \\
& \quad \leqslant k_{1}\left[r_{2}(g x, g u)+r_{2}(g y, g v)\right]+k_{2}\left[r_{2}(g x, F(x, y))+r_{2}(g y, F(y, x))\right]+k_{3}\left[r_{2}(g u, F(u, v))+r_{2}(g v, F(v, u))\right]
\end{aligned}
$$

holds for all $(x, y),(u, v) \in X \times X$. If $F(X \times X) \subset g(X)$ and $g(X)$ is $r_{1}$-complete, then $F$ and $g$ have a coupled coincidence point $(\mathrm{x}, \mathrm{y}) \in \mathrm{X} \times \mathrm{X}$, satisfying that $\mathrm{gx}=\mathrm{F}(\mathrm{x}, \mathrm{y})=\mathrm{gy}=\mathrm{F}(\mathrm{y}, \mathrm{x})$. Moreover, if $\mathrm{F}$ and $\mathrm{g}$ are $w$ compatible, then $\mathrm{F}$ and $\mathrm{g}$ have a unique common coupled fixed point of the form $(\mathrm{u}, \mathrm{u})$, which satisfies that $\mathrm{u}=$ $\mathrm{gu}=\mathrm{F}(\mathrm{u}, \mathrm{u})$.

Remark 2.17. If we take coefficient $s=1$ (for the space $X$ ) in Corollaries 2.2-2.4, 2.6-2.15, then several new results can be obtained in two rectangular metric spaces.

\section{Application to integral equations}

Example 3.1. Let $X=\mathbb{R}$ and define $d: X \times X \rightarrow \mathbb{R}^{+}$as $r_{b}(x, y)=|x-y|^{k}, x, y \in X$, where $k \geqslant 1$. Then $\left(X, r_{b}\right)$ is a rectangular b-metric space with coefficient $s=3^{k-1}$. In fact, the conditions $(R b 1)$ and $(R b 2)$ are clear. Next we show that (Rb3). From the convexity of function $f(x)=x^{k}$ for $x \geqslant 0$ and by Jensen inequality we have

$$
(a+b+c)^{k} \leqslant 3^{k-1}\left(a^{k}+b^{k}+c^{k}\right)
$$

for nonnegative real numbers $a, b, c$. This means that

$$
\begin{aligned}
r_{b}(x, y)=|x-y|^{k} \leqslant(|x-z|+|z-w|+|w-y|)^{k} & \leqslant 3^{k-1}\left(|x-z|^{k}+|z-w|^{k}+|w-y|^{k}\right) \\
& =3^{k-1}\left[r_{b}(x, z)+r_{b}(z, w)+r_{b}(w, y)\right]
\end{aligned}
$$

which implies that $(R b 3)$ holds, hence $\left(X, r_{b}\right)$ is a rectangular b-metric space with coefficient $s=3^{k-1}$.

Example 3.2. Let $X=\mathbb{R}$ and $r_{b 1}, r_{b 2}$ are two rectangular $b$-metrics in $X$ such that

$$
r_{b 1}(x, y)=(x-y)^{2}, r_{b 2}(x, y)=\frac{(x-y)^{2}}{2}, \forall x, y \in X
$$

Define $\mathrm{F}: \mathrm{X} \times \mathrm{X} \rightarrow \mathrm{X}$ and $\mathrm{g}: \mathrm{X} \rightarrow \mathrm{X}$ respectively by

$$
F(x, y)=\frac{x-y}{3}, g x=2 x, \forall x, y \in X .
$$

It is easy to see that $F(X \times X) \subset g(X), g(X)$ is $r_{b 1}$-complete, and $F$ and $g$ are $w$-compatible.

On the other hand, we have

$$
\begin{aligned}
r_{b 1}(F(x, y), F(u, v)) & =(F(x, y)-F(u, v))^{2} \\
& =\left(\frac{x-y}{3}-\frac{u-v}{3}\right)^{2} \\
& =\left(\frac{x-u}{3}+\frac{v-y}{3}\right)^{2} \\
& \leqslant 2\left(\left(\frac{x-u}{3}\right)^{2}+\left(\frac{y-v}{3}\right)^{2}\right)
\end{aligned}
$$




$$
\begin{aligned}
& =\frac{1}{9}\left(\frac{(2 x-2 u)^{2}}{2}+\frac{(2 y-2 v)^{2}}{2}\right) \\
& =\frac{1}{9}\left(\frac{(g x-g u)^{2}}{2}+\frac{(g y-g v)^{2}}{2}\right)=\frac{1}{9}\left[r_{b 2}(g x, g u)+r_{b 2}(g y, g v)\right]
\end{aligned}
$$

By similar arguments as above, we can show that

$$
r_{\mathrm{b} 1}(\mathrm{~F}(\mathrm{y}, z), \mathrm{F}(v, \mathrm{u})) \leqslant \frac{1}{9}\left[\mathrm{r}_{\mathrm{b} 2}(\mathrm{gy}, \mathrm{gv})+\mathrm{r}_{\mathrm{b} 2}(\mathrm{gx}, \mathrm{gu})\right] .
$$

Combining the above two inequalities, we obtain

$$
r_{\mathrm{b} 1}(F(x, y), F(u, v))+r_{b 1}(F(y, x), F(v, u)) \leqslant \frac{2}{9}\left[r_{b 2}(g x, g u)+r_{b 2}(g y, g v)\right] .
$$

Then by Corollary 2.6, $\mathrm{F}$ and $\mathrm{g}$ have a unique common coupled fixed point, in fact, $(0,0)$ is the unique common coupled fixed point of mappings of $\mathrm{F}$ and $\mathrm{g}$.

Next, we assume that $X=C[a, b]$ is the set of all continuous functions. Define two rectangular bmetrics respectively by

$$
r_{b 1}(x, y)=\max _{t \in[a, b]}|x(t)-y(t)|^{k}, \quad r_{b 2}(x, y)=\frac{\max _{t \in[a, b]}|x(t)-y(t)|^{k}}{2}, \forall x, y \in X,(k \geqslant 1) .
$$

Then the coefficient of two rectangular $b$-metrics is $s=3^{k-1}$. Consider the nonlinear integral equation set as follows:

$$
\left\{\begin{array}{l}
x(r)=K(r)+\int_{a}^{b} G(r, t)[f(t, x(t))+g(t, y(t))] d t \\
y(r)=K(r)+\int_{a}^{b} G(r, t)[f(t, y(t))+g(t, x(t))] d t
\end{array}\right.
$$

Next, we will analyze (3.1) under the following conditions:

(i) $f, g:[a, b] \times R \rightarrow R$ are three continuous functions.

(ii) $\mathrm{K}:[\mathrm{a}, \mathrm{b}] \rightarrow \mathrm{R}$ are continuous functions.

(iii) $\mathrm{G}:[\mathrm{a}, \mathrm{b}] \times \mathrm{R} \rightarrow[0,+\infty)$ are continuous functions.

(iv) There exists $p, q>0$ such that for all $x, y \in R$,

$$
\left\{\begin{array}{l}
|f(t, x(t))-f(t, y(t))| \leqslant p|x-y| \\
|g(t, x(t))-g(t, y(t))| \leqslant q|x-y|
\end{array}\right.
$$

(v)

$$
\max _{r \in[a, b]}\left(\int_{a}^{b}|G(r, t)| d t\right)^{k}<\frac{1}{2^{k+1} L^{k}}, \text { with } L=\max \{p, q\} .
$$

Theorem 3.3. Under the conditions (i)-(v), the integral equation (3.1) has a unique common solution on [a, b].

Proof. Define $\mathrm{F}: \mathrm{X} \times \mathrm{X} \rightarrow \mathrm{X}$ and $\mathrm{g}: \mathrm{X} \rightarrow \mathrm{X}$ respectively by

$$
\begin{aligned}
F(x, y)(r) & =K(r)+\int_{a}^{b} G(r, t)[f(t, x(t))+g(t, y(t))] d t, \\
g x & =2 x, \forall x \in X, t \in[a, b], x, y \in X, \\
r_{b 1}(F(x, y), F(u, v)) & =\max _{r \in[a, b]}|F(x, y)(r)-F(u, v)(r)|^{k}, \forall x, y, u, v \in X, \\
r_{b 2}(F(x, y), F(u, v)) & =\frac{\max _{r \in[a, b]}|F(x, y)(r)-F(u, v)(r)|^{k}}{2}, \forall x, y, u, v \in X .
\end{aligned}
$$


It is easy to see that $F(X \times X) \subset g(X), g(X)$ is $r_{b 1}$-complete, and $F$ and $g$ are $w$-compatible.

On the other hand, from (iv) and (v), we have

$$
\begin{aligned}
& |F(x, y)(r)-F(u, v)(r)|^{k} \\
& \quad=\left|\int_{a}^{b} G(r, t)[f(t, x(t))-f(t, u(t))] d t+\int_{a}^{b} G(r, t)[g(t, y(t))-g(t, v(t))] d t\right|^{k} \\
& \quad \leqslant 2^{k-1}\left|\int_{a}^{b} G(r, t)[f(t, x(t))-f(t, u(t))] d t\right|^{k}+2^{k-1}\left|\int_{a}^{b} G(r, t)[g(t, y(t))-g(t, v(t))] d t\right|^{k} \\
& \quad \leqslant 2^{k-1}\left[\left|\int_{a}^{b} G(r, t)[f(t, x(t))-f(t, u(t))] d t\right|^{k}+\left|\int_{a}^{b} G(r, t)[g(t, y(t))-g(t, v(t))] d t\right|^{k}\right] \\
& \quad \leqslant 2^{k-1}\left[p^{k}\left(\max _{t \in[a, b]}|x(t)-u(t)|\right)^{k}+q^{k}\left(\max _{t \in[a, b]}|y(t)-v(t)|\right)^{k}\right] \cdot\left(\int_{a}^{b} G(r, t) d t\right)^{k} \\
& \quad \leqslant 2^{k-1} L^{k}\left[\max _{t \in[a, b]}|x(t)-u(t)|^{k}+\max _{t \in[a, b]}|y(t)-v(t)|^{k}\right] \cdot \frac{1}{2^{k+1} L^{k}} \\
& \quad \leqslant \frac{1}{2^{k+2}}\left[\max _{t \in[a, b]}|2 x(t)-2 u(t)|^{k}+\max _{t \in[a, b]}|2 y(t)-2 v(t)|^{k}\right] \\
& \quad=\frac{1}{2^{k+1}}\left[r_{b 2}(g x, g u)+r_{b 2}(g y, g v)\right] .
\end{aligned}
$$

It follows from the above inequality, we have

$$
r_{b 1}(F(x, y), F(u, v))=\max _{r \in[a, b]}|F(x, y)(r)-F(u, v)(r)|^{k} \leqslant \frac{1}{2^{k+1}}\left[r_{b 2}(g x, g u)+r_{b 2}(g y, g v)\right] .
$$

By similar arguments as above,

$$
r_{b 1}(F(y, x), F(v, u)) \leqslant \frac{1}{2^{k+1}}\left[r_{b 2}(g y, g v)+r_{b 2}(g x, g u)\right] .
$$

It follows from (3.2) and (3.3) that we have

$$
r_{b 1}(F(x, y), F(u, v))+r_{b 1}(F(y, x), F(v, u)) \leqslant \frac{1}{2^{k}}\left[r_{b 2}(g x, g u)+r_{b 2}(g y, g v)\right] .
$$

Consequently, all the conditions of Corollary 2.6 are satisfied. It follows from the result of Corollary 2.6 that $F$ and $g$ have a unique common coupled fixed point $(u, u)$, satisfying $F(u, u)=g u=u$. So $(u, u)$ is the unique solution of integral equation (3.1).

\section{Acknowledgment}

This work is supported by the National Natural Science Foundation of China $(11071169,11271105)$, the Natural Science Foundation of Zhejiang Province (Y6110287).

\section{References}

[1] M. Abbas, M. A. Khan, S. Radenović, Common coupled fixed point theorems in cone metric spaces for w-compatible mappings, Appl. Math. Comput., 217 (2010), 195-202. 1.8

[2] T. Abdeljawad, D. Türkog̃lu, Locally convex valued rectangular metric spaces and the Kannan's fixed point theorem, J. Comput. Anal. Appl., 14 (2012), 484-494. 1 
[3] M. Arshad, J. Ahmad, E. Karapınar, Some common fixed point results in rectangular metric spaces, Int. J. Anal., 2013 (2013), 7 pages. 1

[4] H. Aydi, A. Felhi, S. Sahmim, Common fixed points in rectangular b-metric spaces using (E.A) property, J. Adv. Math. Stud., 8 (2015), 159-169. 1

[5] H. Aydi, E. Karapinar, H. Lakzian, Fixed point results on a class of generalized metric spaces, Math. Sci. (Springer), 2012 (2012), 6 pages. 1, 1

[6] A. Branciari, A fixed point theorem of Banach-Caccioppoli type on a class of generalized metric spaces, Publ. Math. Debrecen, 57 (2000), 31-37. 1, 1.2

[7] S. Czerwik, Contraction mappings in b-metric spaces, Acta Math. Inform. Univ. Ostraviensis, 1 (1993), 5-11. 1, 1.1

[8] C. Di Bari, P. Vetro, Common fixed points in generalized metric spaces, Appl. Math. Comput., 218 (2012), 7322-7325. 1

[9] H.-S. Ding, M. Imdad, S. Radenović, J. Vujaković, On some fixed point results in b-metric, rectangular and b-rectangular metric spaces, Arab J. Math. Sci., 22 (2016), 151-164. 1

[10] İ. M. Erhan, E. Karapınar, T. Sekulić, Fixed points of $(\psi, \phi)$ contractions on rectangular metric spaces, Fixed Point Theory Appl., 2012 (2012), 12 pages. 1

[11] R. George, S. Radenović, K. P. Reshma, S. Shukla, Rectangular b-metric space and contraction principles, J. Nonlinear Sci. Appl., 8 (2015), 1005-1013. 1, 1.3, 1, 1.4, 1

[12] R. George, R. Rajagopalan, Common fixed point results for $\psi-\phi$ contractions in rectangular metric spaces, Bull. Math. Anal. Appl., 5 (2013), 44-52. 1

[13] T. Gnana Bhaskar, V. Lakshmikantham, Fixed point theorems in partially ordered metric spaces and applications, Nonlinear Anal., 65 (2006), 1379-1393. 1.5

[14] H. Isık, D. Türkoğlu, Common fixed points for $(\psi, \alpha, \beta)$-weakly contractive mappings in generalized metric spaces, Fixed Point Theory Appl., 2013 (2013), 6 pages. 1

[15] W. A. Kirk, N. Shahzad, Generalized metrics and Caristi's theorem, Fixed Point Theory Appl., 2013 (2013), 9 pages.

[16] B. K. Lahiri, P. Das, Fixed point of a Ljubomir Ćirić's quasi-contraction mapping in a generalized metric space, Publ. Math. Debrecen, 61 (2002), 589-594. 1

[17] V. Lakshmikantham, L. Ćirić, Coupled fixed point theorems for nonlinear contractions in partially ordered metric spaces, Nonlinear Anal., 70 (2009), 4341-4349. 1.6, 1.7

[18] H. Lakzian, B. Samet, Fixed points for $(\psi, \phi)$-weakly contractive mappings in generalized metric spaces, Appl. Math. Lett., 25 (2012), 902-906. 1

[19] V. La Rosa, P. Vetro, Common fixed points for $\alpha-\psi-\phi$-contractions in generalized metric spaces, Nonlinear Anal. Model. Control, 19 (2014), 43-54. 1

[20] J. R. Roshan, V. Parvaneh, Z. Kadelburg, H. Zoran, New fixed point results in b-rectangular metric spaces, Nonlinear Anal. Model. Control, 21 (2016), 614-634. 1

[21] B. Samet, A fixed point theorem in a generalized metric space for mappings satisfying a contractive condition of integral type, Int. J. Math. Anal. (Ruse), 3 (2009), 1265-1271. 1 